\title{
Determination of the zero-order fringe position in digital speckle pattern interferometry
}

\author{
Rodrigo Henao, Héctor Rabal, Alberto Tagliaferri, and Roberto Torroba
}

\begin{abstract}
A method for determining the position of the zero-order fringe in a metrological experiment with digital speckle pattern interferometry is proposed. It is based on an averaging procedure with shifted images obtained before and after a load is applied. This technique is a complement to the phase-shifting methods. Experimental examples are shown. (c) 1997 Optical Society of America

Key words: Optical metrology, interferometry, speckle, speckle correlation, TV holography.
\end{abstract}

\section{Introduction}

Interferometry can be used to produce a fringe pattern that represents the field surface displacement of an object in response to some change in mechanical loading. ${ }^{1}$

Digital speckle pattern interferometry (DSPI) is one of the most modern techniques for depicting such fringe patterns. It combines real-time processing with the flexibility of software handling. ${ }^{2}$ DSPI was developed by combining the well-known techniques of holographic and speckle interferometry by using an image hologram setup and following the methods of double-exposure holography. It utilizes a CCD camera interfaced to a computer to process the data. For comparison measurements, a reference frame stored in memory is continuously subtracted from incoming data, and then the intensity difference is displayed. The fringes represent the correlation between the speckle patterns. However, for quantitative purposes, interferograms must be analyzed so that the results can be presented in the required numerical form. ${ }^{3}$ Progress has been made in fringe pattern analysis with a number of electronic aids. These devices allowed a substantial improvement in the accuracy of fringe location within an interferogram.

The wide availability of digital image processing equipment prompted a number of studies to investigate different alternatives of automatic interferogram analyzing. Modern techniques have reached a

The authors are with Centro de Investigaciones Opticas, Casilla de Correo 124, 1900 La Plata, Argentina.

Received 11 January 1996; revised manuscript received 21 June 1997.

0003-6935/97/102066-04\$10.00/0

(C) 1997 Optical Society of America point at which they can provide useful results, especially with phase-stepping methods. 4 By introducing discrete shifts in the position of the fringes, we can calculate the phase map at pixel location $(i, j)$ in a relatively simple way. The phase is changed when a mirror with a computer-controlled piezoelectric translator is moved.

The successful interpretation of an interferogram depends to a great extent on the ability to assign the right order numbers to the fringes. This fringe labeling is used to determine the displacement of a point relative to a certain origin when the sensitivity vector of the experiment is known. Order ambiguity is then a major source of errors, both for displacement measurements and for generating and interpreting contour fringes. Analogical ${ }^{5}$ and digital ${ }^{6}$ holographic contouring have used multiple illumination sources to modify the fringe structure as a means for reducing order assignment ambiguity.

Another technique for contouring with multiple digital images is to synthesize a particular fringe profile, namely, that of an approximate delta function. ${ }^{7}$ That fringe corresponds to the zero-order interference fringe, showing the locus of places with a zero optical path difference between the two illuminating beams. Using such a single-fringe projection, we can eliminate order ambiguity.

We present in this study a method for displaying the fringe of zero displacement by using the same principle. Several illuminating beams are provided for that purpose by the movement of a lens controlled by a stepping motor. A series of images are captured and stored with different object illuminations before and after a load is applied to the object. DSPI fringes are obtained by subtracting the corresponding pairs of images, and the results are then incoherently added, resulting in a single dark fringe showing the 
locus where no displacement occurred. Accurate repositioning of the lens at its original starting point before object loading is required for the successful application of the method. If this condition is not fulfilled, the dark fringe blurs out its contrast but does not change its position. Experimental verifications of the proposal are presented.

\section{Background of the Technique}

The basic DSPI equation describing the intensity difference in an image point between a reference frame and the current one is given by ${ }^{8}$

$$
\Delta I=4\left(I_{o} I_{r}\right)^{1 / 2} \sin \left(\varphi+\frac{\delta}{2}\right) \sin \frac{\delta}{2},
$$

where $I_{o}$ and $I_{r}$ are the intensities of the object and the reference beams, respectively, $\varphi$ is the random phase of the speckle pattern produced by surface roughness, and $\delta$ is the phase change caused by the modified optical path produced, for example, by a displacement between exposures of the corresponding object point. In principle, if the optical system is stable, $I_{o}, I_{r}$, and $\varphi$ are constants for a given point. Therefore $\Delta I$ is a function only of $\delta$. For an object point, $P(x, y, z)$, suffering a displacement, $\mathbf{d}=\mathbf{d}(x, y$, $z), \delta$ is given by 9

$$
\frac{\delta(P)}{2 \pi} \lambda=N(P) \lambda=\left[e_{B}(P)+e_{Q}(P)\right] \cdot \mathbf{d}(P)=\mathbf{S}(P) \cdot \overrightarrow{\mathbf{d}}(P),
$$

where $N(P)$ is the number of fringes in the image between a fixed point (a point that suffered no displacement between exposures) and $P, \lambda$ is the light wavelength, $e_{B}$ is a unit vector from $P$ in the observation direction, $e_{Q}$ is a unit vector from $P$ in the illumination direction, and $\mathbf{S}$ is the sensitivity vector. We assume the displacements field to be continuous, and we also suppose that in the image a connected path exists between a fixed point and $P$.

A displacement $\mathbf{d}(P)$ then produces a phase change $\delta$. If it is great enough (corresponding to a phase difference greater than $2 \pi$ ), the intensity $\Delta I$ will lie somewhere between the extreme values $I_{\max }$ and $I_{\min }$. This $I_{\min }$ is zero whenever $\delta \equiv 2 n \pi ; n=0,1$, $2, \ldots$ To construct the phase map, we often require the position of at least one point that suffered no displacement. It is used as an origin for counting fringes to every point of interest. If the sensitivity vector is known, the number of fringes between the origin mentioned and a generic point $P$ determines only one component of the displacement. Three in= dependent equations are required as a minimum for solve for three components of $\mathbf{d}$ :

$$
\begin{aligned}
N_{i}(P) \lambda & =\left[e B(P)+e_{Q_{i}}(P)\right] \cdot \mathbf{d}(P)=\mathbf{S}_{i}(P) \cdot \mathbf{d}(P) \\
i & =1,2, \ldots, n \quad n>2 .
\end{aligned}
$$

Overdetermined systems combined with the leastsquares calculations are usually used to increase the accuracy of the measurement. 9 The sign of every component remains nevertheless ambiguous.

It is evident that the existence of a nondeformed point is not a priori warranted. Whole-field displacements have no such a point. Even if such a point exists, it may be located outside the observation field of the experiment. The phase change $\delta$ is then measured with $2 n \pi$ ambiguity.

When $\mathbf{d}=0, \delta=0$ for all sensitivity vectors. This is the only case that gives a zero phase shift for such general geometries. That is, the $\mathbf{d}=0$ locus will be a dark fringe in DSPI, which is obtained through the subtraction of the before and the after states. The converse is true in analogic holography.

If several (in principle, infinite) sensitivity vectors are used, the only dark fringe in the DSPI image common to all observation points, or illuminations or combinations of both, should correspond to the locus $\mathbf{d}=0$, if it exists. When, as in practice, only a finite number of sensitivity vectors are available, several isolated zero intensity fringes will appear. An $a$ priori knowledge of the expected geometry of the displacements could then be used to decide which represents the zero order.

With the technique we propose that a set of images be stored with a progressive increment in the angle directing the object-beam illumination. This increment is obtained by careful movements of a lens controlled by a stepping motor. The illumination directions must be chosen so that every possible displacement produces a phase change, that is, three sensitivity vectors should constitute a basis. Then the load is applied to the object and a similar set of images is recorded, repeating the same illuminating directions of the object beams as before. For each pixel $(i, j)$ the computer assigns a $z$ value to the resulting image calculated as

$$
z=\sum_{k}\left|\left(z_{k}-z_{k}^{\prime}\right)\right|
$$

where $z_{k}$ is the gray level in the recorded image corresponding to the $k$ th illumination direction before loading, $z_{k}{ }^{\prime}$ is the corresponding value after loading,

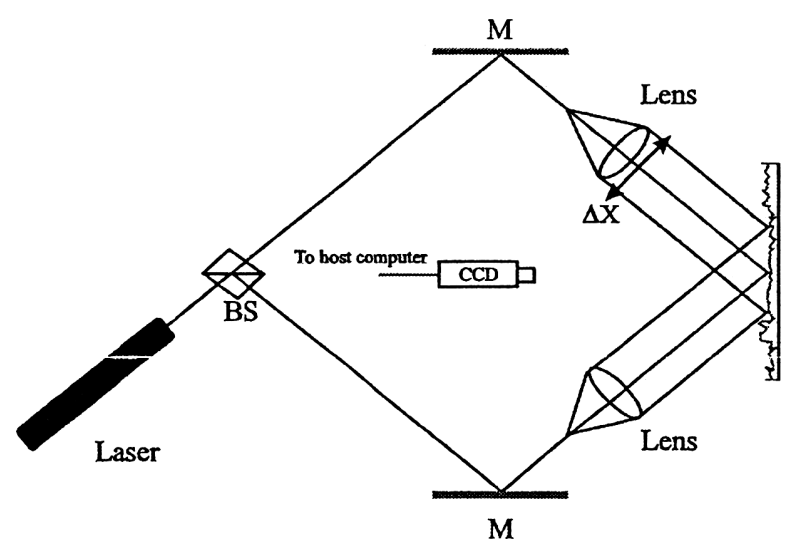

Fig. 1. Experimental configuration used in the technique. 


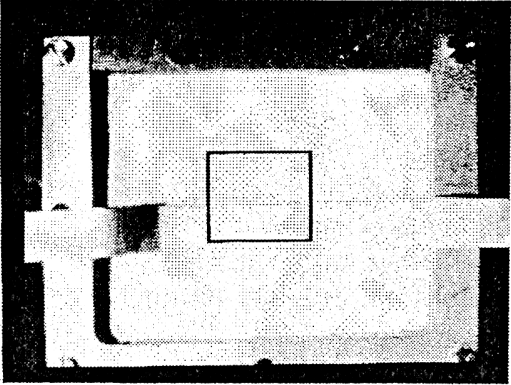

(a)

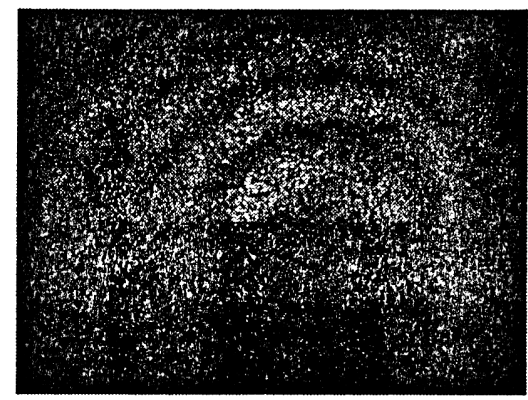

(b)

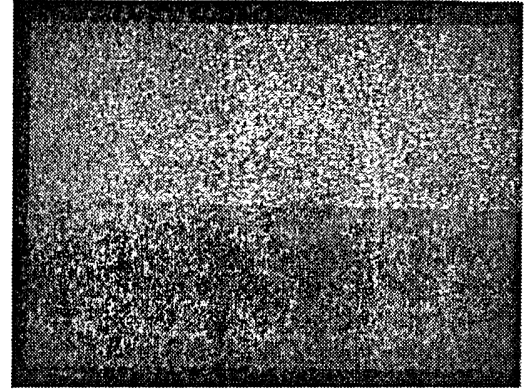

(c)

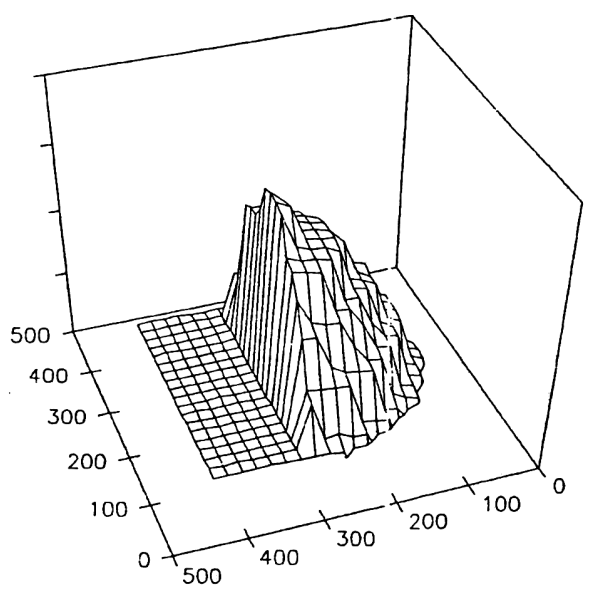

(d)

Fig. 2. (a) Clamped metal plate loaded on its center compared with a fixed metal strip. The rectangle indicates the observation area. (b) DSPI display. Result after (c) the averaging procedure and (d) the phase mapping. The metal strip remains black, and the fringes are removed.

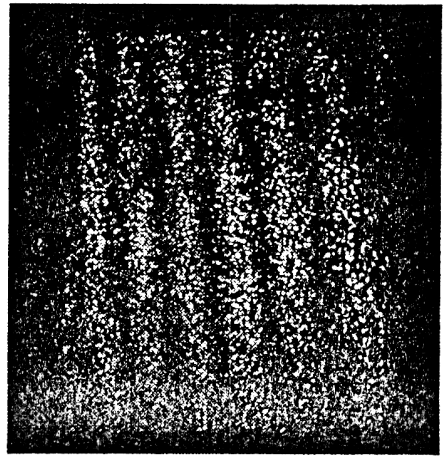

(a)

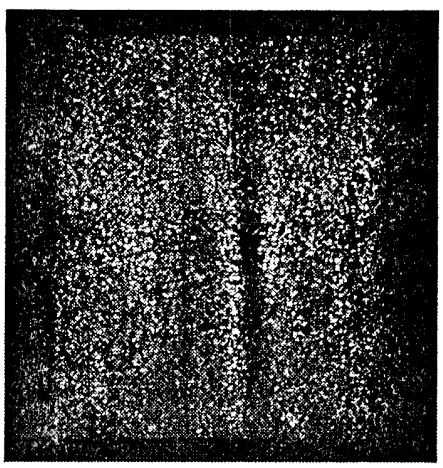

(b)

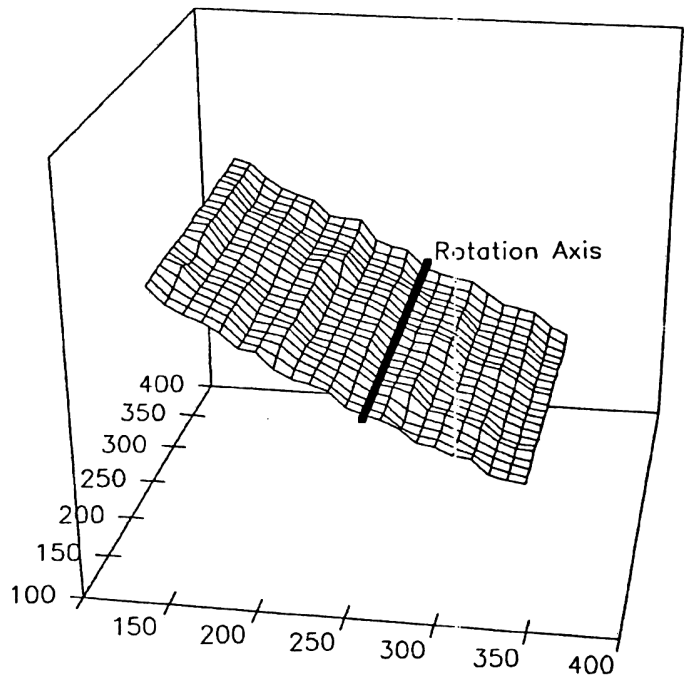

(c)

Fig. 3. Composition showing (a) the fringe resulting from the rotation of a plate, (b) the zero-order fringe (rotational axis) arising from the digital averaging, (c) the phase-mapping obtained from (a) together with the position of the rotational axis obtained from (b).

and the bars indicate absolute value. The zerodisplacement locus does not move during a change in the illumination direction. It plays here the role of the zero-path difference in Ref. 7. Skeletonization of this fringe provides an origin for counting the fringe 
in any of the pairs $z_{k}, z_{k}{ }^{\prime}$. The rest of the fringes move when the illumination direction changes and wash out in the averaging operation.

For a given sensitivity vector there are nonzero displacements that produce a dark fringe in the image, namely, those corresponding to directions perpendicular to that vector. The sensitivity vector set is determined from the observation direction, and the different illumination directions can then be chosen to show a dark fringe corresponding to a subspace of the displacement field, the loci of places that did not move in a certain direction. If, for example, all the sensitivity vectors are contained in a single plane, points with displacements perpendicular to that plane will appear dark in the resulting image. As a consequence, regions obeying a defined property can be highlighted if required for a particular experiment.

\section{Experiments}

Several experiments were conducted to demonstrate the validity of our proposal. Figure 1 shows the experimental setup. A linear polarized $\mathrm{He}-\mathrm{Ne}$ laser beam is divided by the beam splitter, BS. One arm serves as a reference beam, and the other is used to illuminate the object through a collimating lens, which is mounted on a stepping motor. This last introduces the required changes in the illuminating beam direction. In all our experiments five image pairs were stored with a step of $30 \mu \mathrm{m}$ between images.

In the case of Fig. 2 a clamped planar metallic surface, deformed under a load applied from the rear on its center, and an unperturbed metal strip are compared. Figure 2(a) shows the object, and the rectangle indicates the observation area. Figure 2(b) shows the display of the normal DSPI result. The result of applying Eq. (2) to these images is displayed in Fig. 2(c). The metal strip remains totally black, indicating that no deformation occurred to it. The corresponding phase mapping is shown in Fig. 2(d).

In Fig. 3 a plane object is rotated around an axis. In Fig. 3(a) the DSPI interference fringes are shown, whereas Fig. 3(b) shows the actual rotation axis after our procedure is applied. In this example, the axis is the zero-order fringe. Figure $3(\mathrm{c})$ is the result of the phase mapping of Fig. 3(a) and the position of the rotational axis obtained from Fig. 3(b).

A lack of repeatability of positioning between the acquisition of the reference state and deformed state frames may occur. If such an error introduces an extra optical path of less than $\lambda / 2$, no fringe location error appears but a blurring in its contrast. The error depends on the apparatus and the involved setup geometry, but it is not a serious constraint. In our case, $s$, the maximum error was of $0.05 \mu \mathrm{m}$ for the whole experiment. This value can be achieved with commercially available equipment. On the other hand, the geometric parameters, like the focal length or the observation angle, can be adjusted to bring the appropriate value for a particular experiment.

\section{Conclusions}

We have demonstrated the possibility of a zero-order fringe assignment with the aid of DSPI techniques in addition to a multiple-image storing procedure. The proposed method is to some extent complementary with the phase-stepping techniques. Once the zeroorder fringe is localized, we can proceed with the standard phase-mapping methods to find the absolute deformation field distribution. The method reported here is suitable only for static loading.

The financial support of the Consejo Nacional de Investigaciones Científicas y Tecnicas (Argentina) and Conselho Nacional de Desenvolvimiento Cientifico e Tecnologico (Brazil) agreement, the Third World Academy of Science grant 93-389, the Alexander von Humboldt Foundation (Germany), Fundación Antorchas (Argentina), and Mutis Program (Spain) is gratefully acknowledged.

\section{References}

1. R. Jones and C. Wykes, Holographic and Speckle Interferometry (Cambridge U. Press, Cambridge, UK, 1983), Chap. 4, pp. 183188.

2. $\mathrm{K}$. Creath and G. Slettemoen, "Vibration-observation techniques for digital speckle pattern interferometry," J. Opt. Soc. Am. A 2, 1629-1636 (1986).

3. D. W. Robison, "Automatic fringe analysis with a computer image processing system," Appl. Opt. 22, 2169-2176 (1983).

4. D. Robison and D. Williams, "Digital phase stepping speckle interferometry," Opt. Commun. 57, 26-30 (1986).

5. B. P. Hildebrand and K. A. Haines, "Multiple-wavelength and multiple-source holography," J. Opt. Soc. Am. 57, 155-162 (1967).

6. J. Pomarico, R. Arizaga, H. Rabal, and R. Torroba, "Digital holographic contouring," Opt. Commun. 98, 257-260 (1993).

7. R. Arizaga, H. Rabal, M. Trivi, E. Alanis, and G. Romero, "Single fringe contouring," Opt. Commun. 108, 209-213 (1994).

8. R. Henao, H. Rabal, R. Torroba, and A. Tagliaferri, "Display of the temporal evolution of the speckle patterns," Opt. Eng. 35, 63-69 (1996).

9. W. Osten and W. Juptner, "Measurement of displacement vector fields based on high-precision phase measurements," in Interferometry: Techniques and Analysis, G. M. Brown, M. Kujawinska, O. Y. Kwon, and G. T. Reid, eds., Proc. SPIE 1755, 162-173 (1992). 